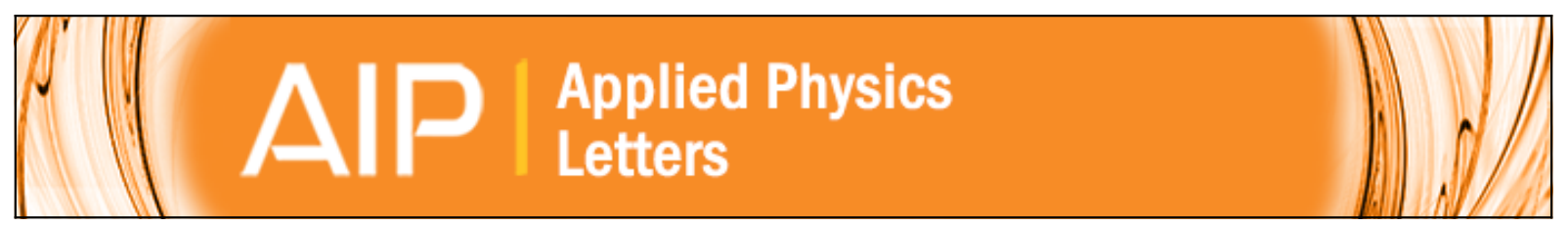

\title{
Broadband negative refractive index obtained by plasmonic hybridization in metamaterials
}

Hien T. Nguyen, Tung S. Bui, Sen Yan, Guy A. E. Vandenbosch, Peter Lievens, Lam D. Vu, and Ewald Janssens

Citation: Applied Physics Letters 109, 221902 (2016); doi: 10.1063/1.4968802

View online: http://dx.doi.org/10.1063/1.4968802

View Table of Contents: http://scitation.aip.org/content/aip/journal/apl/109/22?ver=pdfcov

Published by the AIP Publishing

\section{Articles you may be interested in}

Low-loss negative index metamaterials for $\mathrm{X}, \mathrm{Ku}$, and $\mathrm{K}$ microwave bands

AIP Advances 5, 047119 (2015); 10.1063/1.4918283

Experimental realization of negative refraction using one metasurface

Appl. Phys. Lett. 106, 121903 (2015); 10.1063/1.4916369

Planar metamaterial-based beam-scanning broadband microwave antenna

J. Appl. Phys. 115, 194901 (2014); 10.1063/1.4876233

Design, fabrication, and measurement of highly sub-wavelength double negative metamaterials at high frequencies

J. Appl. Phys. 113, 213712 (2013); 10.1063/1.4809769

Broadband and low loss high refractive index metamaterials in the microwave regime

Appl. Phys. Lett. 102, 091108 (2013); 10.1063/1.4794088

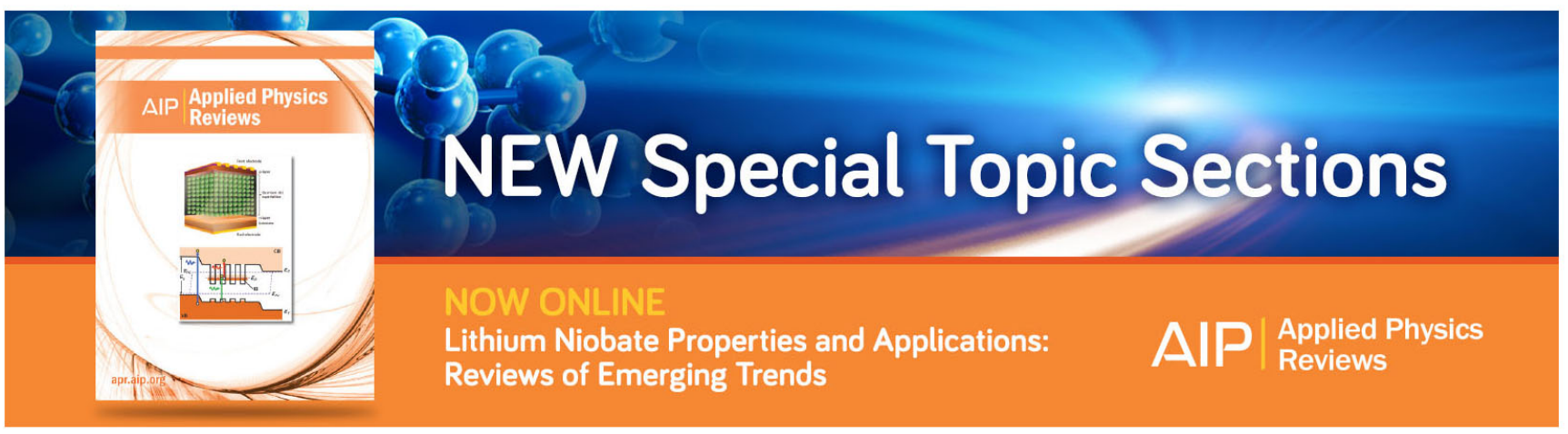




\title{
Broadband negative refractive index obtained by plasmonic hybridization in metamaterials
}

\author{
Hien T. Nguyen, ${ }^{1,2}$ Tung S. Bui, ${ }^{1}$ Sen Yan, ${ }^{3}$ Guy A. E. Vandenbosch, ${ }^{3}$ Peter Lievens, ${ }^{4}$ \\ Lam D. Vu, ${ }^{1, a)}$ and Ewald Janssens ${ }^{4, a)}$ \\ ${ }^{1}$ Institute of Materials Science, Vietnam Academy of Science and Technology, 100000 Hanoi, Vietnam \\ ${ }^{2}$ Thai Nguyen University of Science, 250000 Thai Nguyen, Vietnam \\ ${ }^{3}$ Department of Electrical Engineering, TELEMIC group, KU Leuven, 3001 Leuven, Belgium \\ ${ }^{4}$ Laboratory of Solid State Physics and Magnetism, KU Leuven, 3001 Leuven, Belgium
}

(Received 22 August 2016; accepted 11 November 2016; published online 29 November 2016)

\begin{abstract}
We experimentally demonstrate a broadband negative refractive index (NRI) behavior in combined dimer and fishnet dimer metamaterials operating in the $\mathrm{GHz}$ frequency range. The observations can be well explained by a hybridization model and are in agreement with numerical modelling results. Hybridization of the magnetic resonances is obtained by reducing the distance between the layers in the dimer structures. A ratio of the double negative refractive index bandwidth to operational frequency of approximately $10 \%$ was achieved in the fishnet dimer. The applicable frequency range of the broadband NRI was shown to scale with the size of the structures from the microwave to the far infrared. Published by AIP Publishing. [http://dx.doi.org/10.1063/1.4968802]
\end{abstract}

Negative refractive index (NRI) materials have taken a central role in the development of metamaterials (MM) because of their wide applicability in (opto)electronic devices including superlenses, ${ }^{1}$ antennas, ${ }^{2}$ filters, ${ }^{3}$ resonators, ${ }^{4}$ invisibility cloaks, ${ }^{5}$ and biosensors. ${ }^{6}$ NRI MMs are essentially artificial composites of electric and magnetic structures that have a negative permittivity and negative permeability in the same frequency range. Commonly used artificial composites combine continuous metal wires ${ }^{7}$ with magnetic resonances in split-ring resonators $(\mathrm{SRRs})^{8}$ or cut-wire pairs (CWPs). ${ }^{9-11}$ A major drawback of those conventional structures is that the NRI bandwidth is too narrow to be useful for most practical applications. It was demonstrated that the broadband negative permittivity can be achieved by employing the low-frequency plasma of a continuous wire medium. ${ }^{7,12-14}$ Broadband negative permeability can be obtained by combining individual magnetic resonators with slightly different resonance frequencies. ${ }^{12,15-17}$ That approach is technically challenging as it requires rigorous geometrical optimization. Broadband negative permeability and negative refractive index materials can also be obtained using geometric optimization based on Gielis' superformula. ${ }^{18,19}$ However, the optimal structures have complex shapes, which make them difficult to manufacture. A more convenient way to create a broadband negative permeability and pave the way for practical implementations is using the hybridization of interacting identical resonators. ${ }^{20,21}$

In this letter, the left-handed behavior of two metamaterials, the combined dimer (CD) and the fishnet dimer (FD), is investigated experimentally in the $\mathrm{GHz}$ range. The term dimer refers to a configuration consisting of two identical monomers that are separated by an air gap. ${ }^{21}$ Each monomer is composed of metallic structures that are separated by a dielectric spacer. It is demonstrated that hybridization in

\footnotetext{
a) Authors to whom correspondence should be addressed. Electronic addresses: lamvd@ims.vast.ac.vn, Tel.: +84 4 37917951, Fax: +84 438360705 and ewald.janssens@kuleuven.be, Tel.: +32 16 327207, Fax.: +32 16327983.
}

simple CD and FD structures can be tuned by the interlayer separation and can lead to a significant broadening of the $\mathrm{GHz}$ resonance features. The observed phenomena can qualitatively be explained by the hybridization model and are in quantitative agreement with the numerical modelling results.

Transmission measurements in free space are performed in the $11-17 \mathrm{GHz}$ frequency range using a Hewlett-Packard E8362B network analyzer, connected to microwave standardgain horn antennas. ${ }^{22,23}$ The samples are kept in balance between the horn antennas by a glass sample holder, which is transparent in the frequency range of interest. The measurement setup is constructed to minimize reflections from the surrounding and the sample holder, but reflections on the sample surface itself cannot be avoided. To reduce the effect of interference between the direct and the reflected waves, individual transmission measurements for different positions of the sample relative to the horn antennas are averaged. The distance between the antennas is kept constant at $30 \mathrm{~cm}$, while the sample position is varied in steps of $2 \mathrm{~mm}$ from $14 \mathrm{~cm}$ to $16 \mathrm{~cm}$.

Figure 1 shows the structural parameters of the unit cells and photos of the fabricated CD and FD metamaterials. Each unit cell consists of two monomers separated by a distance $d$. The monomers in the CD structure consists of a CWP and continuous wires, while the fishnet monomer combines slabs with continuous wires. In both monomers, the dielectric layer is made of the composite material FR-4 with a dielectric constant of 4.3 and a loss tangent of 0.025 . The metallic structures are made of copper. Electromagnetic simulations are carried out with finite integration techniques as implemented in CST Microwave Studio, ${ }^{24}$ applying periodic boundary conditions in the $x$ and $y$ directions. Permittivity, permeability, and refraction index $(\varepsilon, \mu, n)$ were calculated from simulated transmission and reflection curves using the retrieval method proposed by Chen et al. ${ }^{25}$

Broadband negative permittivity is rather straightforwardly achieved for frequencies below the plasma frequency of the continuous wires. This plasma frequency, and thus the 

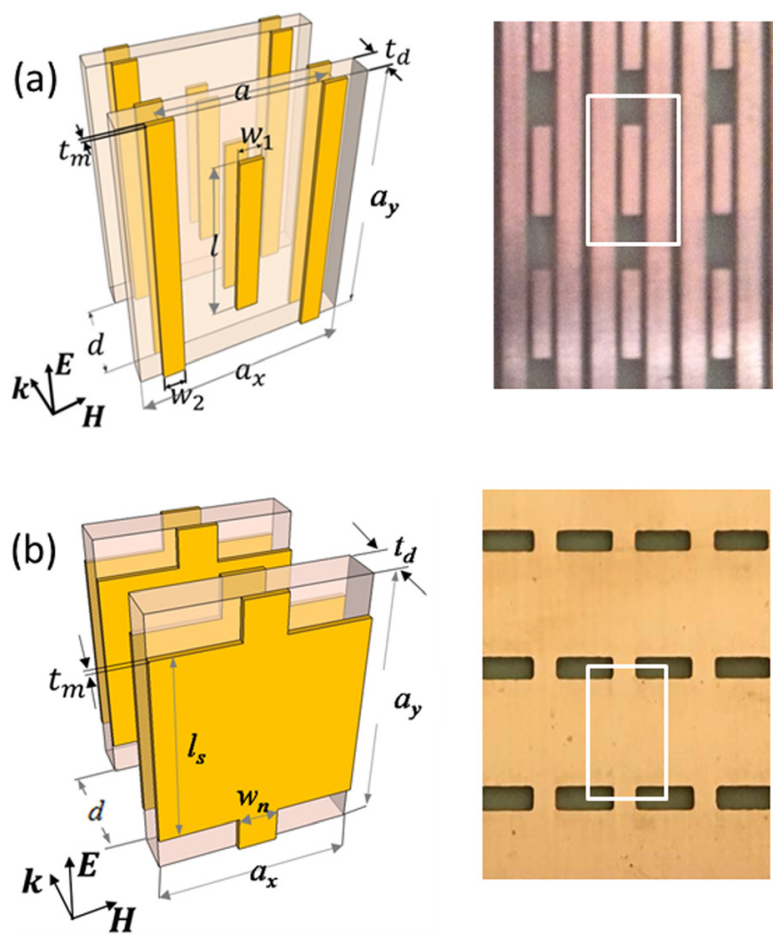

FIG. 1. Scheme of (a) the combined dimer and (b) the fishnet dimer with definitions of the structural parameters and polarization of the electromagnetic wave (left). Images of the fabricated samples taken in the k-direction (i.e., perpendicular to the sample plane) are given on the right. White boxes define the unit cells in that plane. Layer thicknesses for both the CD and the FD are $t_{d}=0.8 \mathrm{~mm}$ and $t_{m}=0.036 \mathrm{~mm}$, while the parameter $d$ was tuned in the $0.8-3.2 \mathrm{~mm}$ range. Other structural parameters are $a_{x}=4 \mathrm{~mm}, a_{y}$ $=8 \mathrm{~mm}, 1=5.5 \mathrm{~mm}$, and $w_{1}=w_{2}=1.0 \mathrm{~mm}$ for the CD and $a_{x}=7 \mathrm{~mm}, a_{y}$ $=9.5 \mathrm{~mm}, w_{n}=1.5$, and $l_{s}=7.0 \mathrm{~mm}$ for the FD.

negative permittivity range, can be tuned by the distance between the continuous wires $a$ and the width of the wires $w_{2}{ }^{7}$ Achieving a broadband negative permeability is more difficult, but can be realized by second-order hybridization as explained below. The total electromagnetic response of a single CWP can be regarded as a hybridization between the surface plasmons in the two individual CWs. This coupling results in two new plasmonic modes: the symmetric $\left|\mathrm{w}_{+}\right\rangle$ and the anti-symmetric $\left|\mathrm{w}_{-}\right\rangle$mode, corresponding to inphase and out-of-phase charge oscillations in the two cut wires, respectively. The symmetric mode effectively operates as two electric dipoles, while the anti-symmetric mode behaves as a magnetic resonance. We therefore focus on the $\left|\mathrm{w}_{-}\right\rangle$mode to achieve broadband negative permeability. The electromagnetic response of a CWP dimer can, analogously, be described by second-order hybridization. ${ }^{20,21}$ If two CWPs are close to each other, interaction between the degenerated $\left|\mathrm{w}_{-}\right\rangle$modes leads to $\left|\mathrm{w}_{--}\right\rangle$and $\left|\mathrm{w}_{-+}\right\rangle$modes. For transversely ordered electrical dipoles, the lower frequency $\left|\mathrm{w}_{--}\right\rangle$and higher frequency $\left|\mathrm{w}_{-+}\right\rangle$are associated with second-order symmetric and antisymmetric modes, respectively. The spacer thickness $t_{d}$ determines the internal coupling between the cut wires within a CWP, while the distance $d$ controls the external interaction strength among the CWPs. Hence, tuning the ratio of $d$ to $t_{d}$ allows manipulating the effectiveness of the second-order hybridization.

To maximize the bandwidth of NRI, we adjusted the structural parameter $d$. Alternatively one could manipulate $t_{d}$ or both $d$ and $t_{d}$. However, previous work showed that both the magnetic resonance frequency and the plasma frequency depend strongly on $t_{d} \cdot{ }^{26}$ It is therefore difficult to adjust the negative permeability and permittivity bands independently by variation of $t_{d}$. The experimental and simulated transmission spectra of the CD metamaterials are summarized in Figs. 2(a) and 2(b), respectively. Both the experiments and the simulations show that there is only one transmission maximum, corresponding to a negative refractive index around $15.4 \mathrm{GHz}$, if the two layers are far away from each other $(d=3.2 \mathrm{~mm})$. Such transmission maximum, or pass band, is a well-known feature of CD metamaterials and its
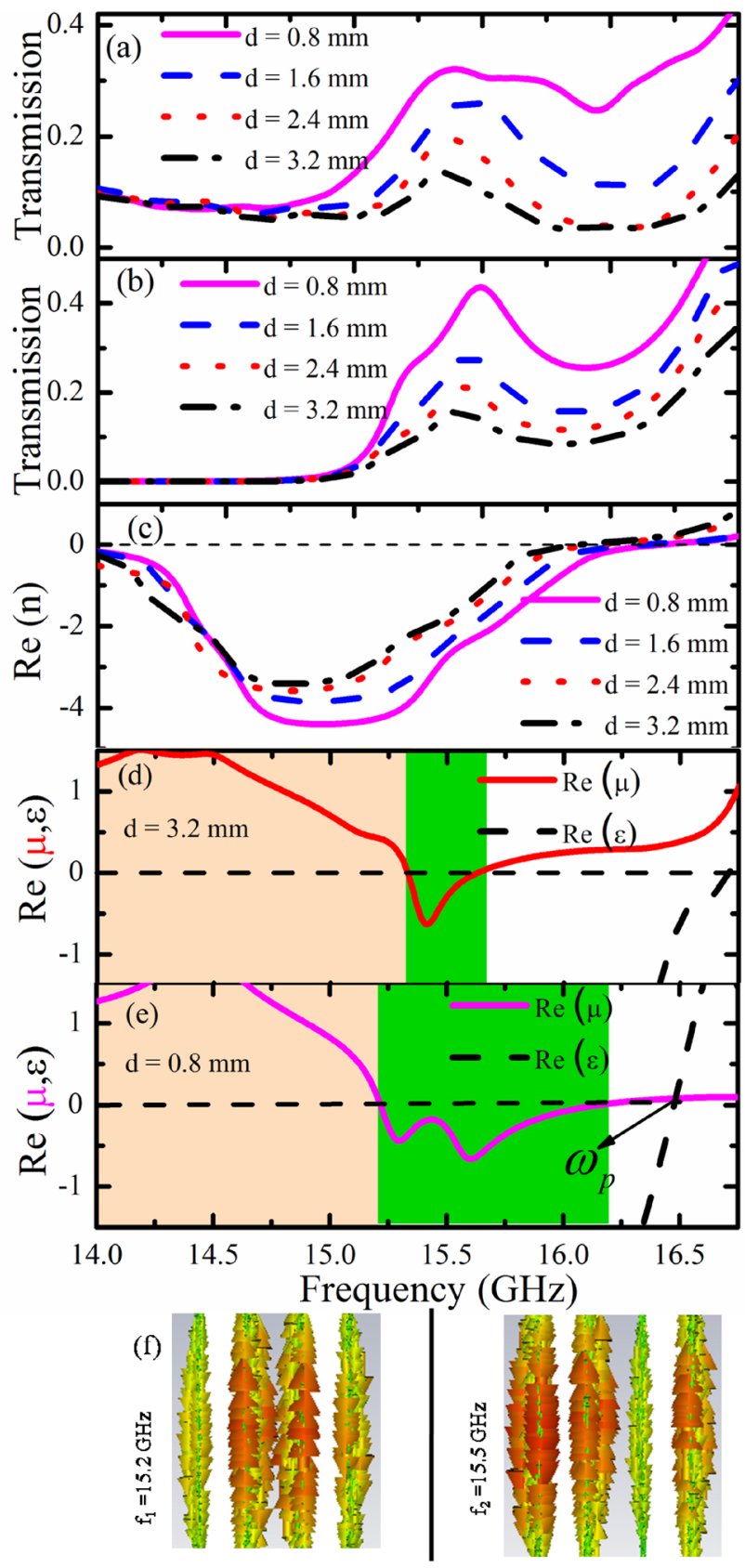

FIG. 2. (a) Experimental and (b) simulated transmission spectra of the CD metamaterials for different distances among the layers $d$. (c) simulated real part of the refractive index, demonstrating a broadband NRI that increases with decreasing $d$. Real part of the permittivity $\varepsilon$ and the permeability $\mu$ for (d) $d=3.2 \mathrm{~mm}$ and (e) $d=0.8 \mathrm{~mm}$. In (d) and (e), the double NRI range is dark shaded (green online), while the single NRI range is light shaded (pink online). (f) Simulated current distributions for the $d=0.8 \mathrm{~mm}$ case at 15.2 and $15.5 \mathrm{GHz}$. 
frequency coincides with a stop band (transmission minimum) of the corresponding CWP. ${ }^{27}$ By decreasing $d$, the transmission peak gradually becomes wider, and finally (for $d=0.8 \mathrm{~mm}$ ) splits into two transmission maxima (around 15.2 and $15.5 \mathrm{GHz}$ ). Current distributions show ring currents at both transmission maxima (see Fig. 2(f)), which demonstrates that they are of magnetic origin. The ring currents in the monomers either have the opposite (lowest energy mode) or the same (highest energy mode) directions. This energetic ordering is the result of a competition between the coupling of the electric and magnetic dipoles. ${ }^{28}$ In a transverse configuration, the lowest energy state corresponds to antiparallel oriented dipoles. In the $15.2 \mathrm{GHz}$ state, magnetic dipoles are antiparallel, while electric dipoles of the middle two cut wires are parallel. Magnetic dipoles in the $15.5 \mathrm{GHz}$ state are parallel, while electric dipoles of all neighboring cut wires are antiparallel. So the magnetic interaction seems to dominate in this case.

The higher simulated transmission of the local maxima at 15.5 GHz, compared to that at $15.2 \mathrm{GHz}$, can be explained by magnetic energy distributions, which suggests that the energy loss is higher at the lower magnetic resonance frequency (not shown here). This prediction could not be resolved in the experimental data. The low transmission at the local maximum (around 35\% in the experiments and $40 \%$ in the simulation) can be explained by the high loss in the FR4 dielectric. Using low loss dielectrics, the resonant splitting is more pronounced and the transmission is higher (simulations predict more than $70 \%$ transmission with Rogers dielectric layers).

The broadened transmission range corresponds to an extended NRI region as illustrated by the simulated real part of the refractive index (Fig. 2(c)). The real parts of permeability and permittivity are shown in Figs. 2(d) and 2(e) for $d=3.2 \mathrm{~mm}$ and $d=0.8 \mathrm{~mm}$, respectively. The evolution of the negative permeability is consistent with the proposed second-order hybridization scheme. For large $d$ values, the $\mathrm{CD}$ exhibits a traditional single magnetic resonance due to the weak coupling between the two layers. When the distance between the layers is reduced, the coupling becomes stronger and the second-order hybridization is activated.

Since the refractive index is a complex value, NRI can be classified either as a single NRI, in which either the real part of the permittivity or the real part of the permeability is negative or as double NRI, in which both the real parts of permittivity and permeability are negative. ${ }^{29}$ As shown in Figs. 2(c)-2(e), the NRI is a little broader after hybridization, but especially the double NRI band, which is most useful for applications, is widened effectively by the interaction among the layers. Indeed, the double NRI bandwidth increases from $0.3 \mathrm{GHz}$ (for $d=3.2 \mathrm{~mm}$ ) to $0.8 \mathrm{GHz}$ (for $d=0.8 \mathrm{~mm}$ ), that is an increase by a factor of 2.5 . For $d=0.8 \mathrm{~mm}$, the ratio of the double NRI bandwidth to the operating frequency is approximately $5 \%$.

In the next step, the expansion of the NRI based on second-order hybridization was investigated for FD structures. The fishnet is a modification of the combined design that arises when the CWs are laterally expanded and merge with the continuous wires. Fishnet structures are easy to design and have less adjustable parameters than combined structures. In addition they provide a good impedance match with free space and thus high transmission values, even when the magnetic-resonance frequency lies far below the effective plasma frequency. ${ }^{30}$

Figures 3(a) and 3(b) are the experimental and simulated transmission spectra of the FD for different $d$ values. The spectra show a pronounced resonance feature around $12 \mathrm{GHz}$. The local maximum in the measured spectra around $11.3 \mathrm{GHz}$ originates from the experimental setup. Its intensity depends on the position of the sample relative to the horn antenna's and therefore is likely caused by superposition of the direct wave with multiple reflected waves between source and sample. Aside from this difference, the simulated and experimental results are in excellent agreement. Similar to the CD, the interaction among the layers becomes stronger with decreasing $d$. This implies an expansion of the NRI range, as shown in Fig. 3(c). The mechanism of the expansion and the classification of the NRI is elucidated by the spectral shape of the real parts of permeability and permittivity (Figs. 3(d) and 3(e)). Obviously the negative permeability band is considerably broadened by reducing $d$.

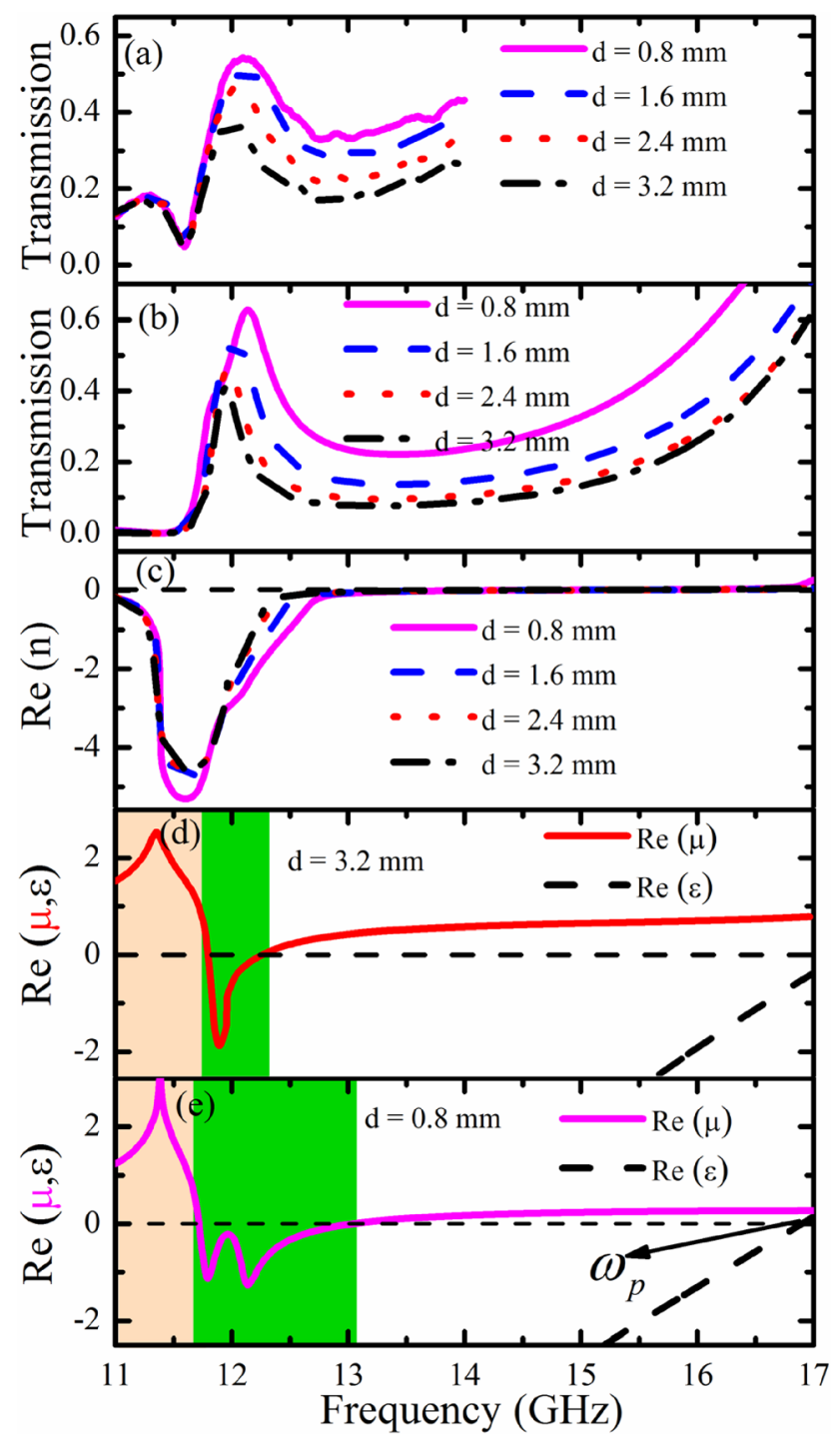

FIG. 3. (a) Experimental and (b) simulated transmission spectra of the FD metamaterials for different distances $d$. (c) simulated real part of the refractive index. Real part of the permittivity $\varepsilon$ and the permeability $\mu$ for (d) $d=3.2 \mathrm{~mm}$ and (e) $d=0.8 \mathrm{~mm}$. In (d) and (e), the double NRI range is dark shaded (green online), while the single NRI range is light shaded (pink online). 


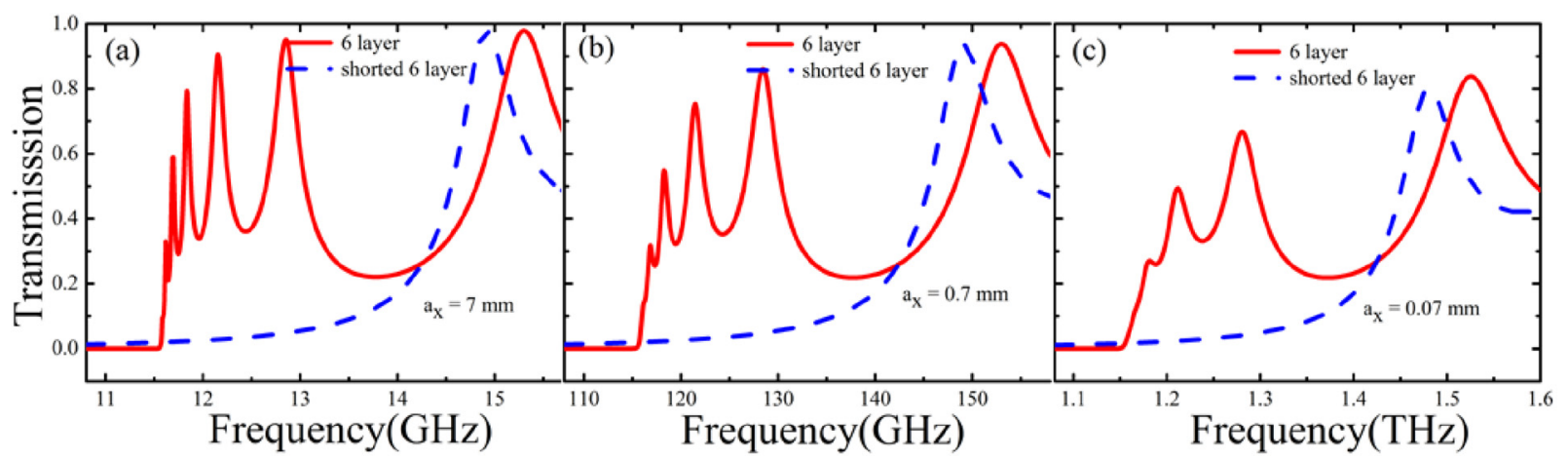

FIG. 4. Simulated transmission of fishnet hexamer and shorted fishnet hexamer corresponding to the lattice constant $a_{x}$ of (a) $7 \mathrm{~mm}$, (b) $0.7 \mathrm{~mm}$, and (c) $0.07 \mathrm{~mm}$.

Different from the $\mathrm{CD}$, the FD transmission exhibits a clear separation of the negative refractive (left-handed) peak from the nearby positive refractive (right-handed) peak, which is advantageous to select the double NRI region. The separations between the two peaks are approximately $1.5(\mathrm{CD})$ and $5 \mathrm{GHz}(\mathrm{FD})$.

The observed Drude-like electric response of the FD $(\varepsilon(\omega)$ in Figs. 3(d) and 3(e)) varies slowly, allowing a good impedance match in a wide frequency range below the plasma frequency. This in turn enhances the transmission, since reflection is reduced when matching the wave impedance, $z=\sqrt{\mu / \varepsilon}$, in the MM to the free space value $z=1$. The dispersion of $\varepsilon(\omega)$ in the CD is more steep (see Figs. 2(d) and 2(e)), which imposes tight constraints to the optimization of the magnetic resonance frequency in conjunction with a good impedance match. The less steep dispersion of the FD permittivity explains why the FD exhibits a higher transmission (above 50\% in the experiments and more than $60 \%$ in the simulations) than the $\mathrm{CD}$, while using the same FR-4 dielectric. Moreover, the ratio of optimum double NRI bandwidth of the FD to its operating frequency is approximately $10 \%$, which is nearly twice as broad as that of the CD structure. This proves that the FD is more suited than the CD to expand the NRI. The current double NRI bandwidth in the $\mathrm{GHz}$ frequency range has a magnitude comparable to that obtained computationally in the visible range for much more complex, and thus more difficult to fabricate, structures. ${ }^{17,19,31}$ The advantages of multiple stacked layers of fishnet structures have been investigated before, ${ }^{30}$ though without assessing the expansion of the NRI bandwidth and the involved mechanisms.

Lastly, electromagnetic simulations were used to investigate the possible extension of the hybridization approach using multilayer fishnet structures. Recent work showed that the response of a $N$-mer MM system can be explained by the hybridization of a monomer with a $(N-1)-m e{ }^{21}{ }^{21}$ Specifically, fishnet hexamer $(\mathrm{FH})$ configurations, consisting of six fishnet structures, were simulated by assuming lossless dielectric and keeping the space between the fishnet monomers fixed at $d=0.8 \mathrm{~mm}$. Also shorted $\mathrm{FH}$, in which neighboring fishnet monomers are shorted at the two ends of the slabs, were investigated. In these shorted structures, the magnetic resonances are absent, which is helpful to identify the double NRI region of the FH. The approach of scaling all geometrical parameters was followed to change the operational frequency range. ${ }^{21}$ The simulated transmission of the $\mathrm{FH}$ and the shorted FH for different dimensions are presented in Fig. 4. A significantly broadened double NRI range is obtained in the FH. The NRI range is scalable from the $\mathrm{GHz}$ to the $\mathrm{THz}$ range when all the geometrical parameters are simultaneously scaled by factors of 0.1 and 0.01 . First the structural parameters of the FD are used to form the FH MM $\left(a_{\mathrm{x}}=7.0 \mathrm{~mm}\right)$, which gives a double NRI region around $12.5 \mathrm{GHz}$. The ratio of the double NRI bandwidth to the operational frequencies is increased from $10 \%(\mathrm{FD})$ to about $12 \%(\mathrm{FH})$. Then, the structures are scaled down by a factor ten $\left(a_{\mathrm{x}}=0.7 \mathrm{~mm}\right)$, which leads to a double NRI region around $125 \mathrm{GHz}$ with a width of $15 \mathrm{GHz}$. The resonance can be moved further to the far-infrared range by reducing the dimensional parameters to the micrometer scale $\left(a_{\mathrm{x}}=0.07 \mathrm{~mm}\right)$. In this case the bandwidth of double NRI is $0.15 \mathrm{THz}$ (from 1.15 to $1.3 \mathrm{THz}$ ). Although the results in Fig. 4 show a linear relation between the NRI region and the scaling factor, the linearity must break down for frequencies above $100 \mathrm{THz}$ due to the free electron kinetic energy. ${ }^{21,32,33}$ This is also forewarned by the transmission of the FH at resonance, which becomes lower when the NRI region shifts from the $\mathrm{GHz}$ to the $\mathrm{THz}$ range. Higher frequencies are more challenging not only because of dissipation owing to the increased optical losses, but also because the scaled-down geometrical parameters may challenge state-ofthe-art nanofabrication technologies.

In conclusion, plasmonic interaction effects were presented to broaden the NRI range in CD and FD metamaterials. Hybridization of the magnetic resonances of the individual layers was achieved by reducing the distance $d$ between the two layers. In case of strongly activated hybridization in the $\mathrm{CD}$, the double NRI range was found to be a factor of 2.5 wider than the non-activated case, and the ratio of the double NRI bandwidth to the operational frequency is approximately $5 \%$. The ratio of the broadband double NRI to the operational frequency could be enhanced further to $10 \%$ by using the FD instead of the CD. At the same time, the transmission of the FD is higher because of a better wave impedance match. Finally, the applicable frequency range of the broadband NRI was shown to be scalable from the microwave to the far infrared via the dimensions of the unit cells and the width of the NRI could be further enhanced by multilayered fishnet structures. This work provides strategies to broaden the NRI bandwidths, which is a central issue in MM research and their use in future devices. 
This research was funded by the Vietnam National Foundation for Science and Technology Development (NAFOSTED) under Grant No. 103.02-2015.84, the Ministry of Education and Training in VietNam under No. B2015TN05-01 and the Belgian Research Foundation-Flanders (FWO) under Grant No. G.A018.12N.

${ }^{1}$ J. B. Pendry, "Negative refraction makes a perfect lens," Phys. Rev. Lett. 85, 3966 (2000).

${ }^{2}$ N. Engheta, "An idea for thin subwavelength cavity resonators using metamaterials with negative permittivity and permeability," IEEE Antennas Wireless Propag. Lett. 1, 10 (2002).

${ }^{3}$ M. Hajizadegan, V. Ahmadi, and M. Sakhdari, "Design and analysis of ultrafast and tunable all optical metamaterial switch enhanced by metal nanocomposite," J. Lightwave Technol. 31, 1877 (2013).

${ }^{4}$ J. Ha, K. Kwon, Y. Lee, and J. Choi, "Hybrid mode wideband patch antenna loaded with a planar metamaterial unit cell," IEEE T. Antenn. Propag. 60, 1143 (2012).

${ }^{5}$ D. Schurig, J. J. Mock, B. J. Justice, S. A. Cummer, J. B. Pendry, A. F. Starr, and D. R. Smith, "Demonstration of a metamaterial electromagnetic cloak at microwave frequencies," Science 314, 977 (2006).

${ }^{6}$ H.-J. Lee and J.-G. Yook, "Biosensing using split-ring resonators at microwave regime," Appl. Phys. Lett. 92, 254103 (2008).

${ }^{7}$ J. B. Pendry, A. J. Holden, W. J. Stewart, and I. Youngs, "Extremely low frequency plasmons in metallic mesostructures," Phys. Rev. Lett. 76, 4773 (1996). ${ }^{8}$ J. B. Pendry, A. J. Holden, D. J. Robbin, and W. J. Stewart, "Magnetism from conductors and enhanced nonlinear phenomena," IEEE Trans. Microwave Theory Tech. 47, 2075 (1999).

${ }^{9}$ A. Ourir and H. H. Ouslimani, "Negative refractive index in symmetric cut-wire pair metamaterial," Appl. Phys. Lett. 98, 113505 (2011).

${ }^{10}$ V. D. Lam, J. B. Kim, S. J. Lee, Y. P. Lee, and J. Y. Rhee, "Dependence of the distance between cut-wire-pair layers on resonance frequencies," Opt. Express 16, 5934 (2008).

${ }^{11} \mathrm{H}$. Wakatsuchi and C. Christopoulos, "Generalized scattering control using cut-wire-based metamaterials," Appl. Phys. Lett. 98, 221105 (2011).

${ }^{12}$ D. H. Kwon, D. H. Werner, A. V. Kildishev, and V. M. Shalaev, "Nearinfrared metamaterials with dual-band negative-index characteristics," Opt. Express 15, 1647 (2007).

${ }^{13}$ C. Huang, Z. Zhao, Q. Feng, J. Cui, and X. Luo, "Metamaterial composed of wire pairs exhibiting dual band negative refraction," Appl. Phys. B 98, 365 (2010).

${ }^{14}$ V. D. Lam, J. B. Kim, S. J. Lee, and Y. P. Lee, "Left-handed behavior of combined and fishnet structures,” J. Appl. Phys. 103, 033107 (2008).

${ }^{15}$ C. Hu, L. Liu, X. N. Chen, and X. G. Luo, "Expanding the band of negative permeability of a composite structure with dual-band negative permeability," Opt. Express 16, 21544 (2008).

${ }^{16}$ Z. Wei, Y. Cao, J. Han, C. Wu, Y. Fan, and H. Li, "Broadband negative refraction in stacked fishnet metamaterial," Appl. Phys. Lett. 97, 141901 (2010).
${ }^{17}$ A. C. Atre, A. García-Etxarri, H. Alaeian, and J. A. Dionne, "A broadband negative index metamaterial at optical frequencies," Adv. Opt. Mater. 1, 327 (2013).

${ }^{18}$ J. Gielis, "A generic geometric transformation that unifies a wide range of natural and abstract shapes," Am. J. Bot. 90, 333 (2003).

${ }^{19}$ S. Zhou, S. Townsend, Y. M. Xie, X. Huang, J. Shen, and Q. Li, "Design of fishnet metamaterials with broadband negative refractive index in the visible spectrum," Opt. Lett. 39, 2415 (2014).

${ }^{20}$ N. T. Tung, D. T. Viet, B. S. Tung, N. V. Hieu, P. Lievens, and V. D. Lam, "Broadband negative permeability by hybridized cut-wire pair metamaterials," Appl. Phys. Express 5, 112001 (2012).

${ }^{21}$ N. T. Tung, B. S. Tung, E. Janssens, P. Lievens, and V. D. Lam, "Broadband negative permeability using hybridized metamaterials: Characterization, multiple hybridization, and terahertz response," J. Appl. Phys. 116, 083104 (2014).

${ }^{22}$ V. Volski, G. A. E. Vandenbosch, and A. Vasylchenko, "A dedicated technique to measure shielding effectiveness of textiles using a two horn antenna set-up,” J. Text. Inst. 102, 164 (2011).

${ }^{23}$ S. Yan and G. A. E. Vandenbosch, "Compact circular polarizer based on chiral twisted double split-ring resonator," Appl. Phys. Lett. 102, 103503 (2013).

${ }^{24}$ See www.cst.com for Computer Simulation Technology Studio Suite.

${ }^{25}$ X. Chen, T. M. Grzegorczyk, B. I. Wu, J. Pacheco, Jr., and J. A. Kong, "Robust method to retrieve the constitutive effective parameters of metamaterials," Phys. Rev. E 70, 016608 (2004).

${ }^{26}$ V. D. Lam, N. T. Tung, M. H. Cho, J. W. Park, W. H. Jang, and Y. P. Lee, "Effect of the dielectric layer thickness on the electromagnetic response of cut-wire-pair and combined structures," J. Phys. D: Appl. Phys. 42, 115404 (2009).

${ }^{27}$ T. T. Nguyen, P. Lievens, Y. P. Lee, and D. L. Vu, "Computational studies of a cut-wire pair and combined metamaterials," Adv. Nat. Sci.: Nanosci. Nanotechnol. 2, 033001 (2011).

${ }^{28}$ N. Liu and H. Giessen, "Coupling effects in optical metamaterials," Angew. Chem. Int. Ed. 49, 9838 (2010).

${ }^{29}$ N. T. Tung, V. D. Lam, J. W. Park, M. H. Cho, Y. P. Lee, J. Y. Rhee, and W. H. Jang, "Single- and double-negative refractive indices of combined metamaterial structure," J. Appl. Phys. 106, 053109 (2009).

${ }^{30}$ M. Kafesaki, I. Tsiapa, N. Katsarakis, Th. Koschny, C. M. Soukoulis, and E. N. Economou, "Left-handed metamaterials: The fishnet structure and its variations," Phys. Rev. B 75, 235114 (2007).

${ }^{31}$ G. Dolling, M. Wegener, C. M. Soukoulis, and S. Linden, "Negative-index metamaterial at $780 \mathrm{~nm}$ wavelength," Opt. Lett. 32, 53 (2007).

${ }^{32}$ R. S. Penciu, M. Kafesaki, Th. Koschny, E. N. Economou, and C. M. Soukoulis, "Magnetic response of nanoscale left-handed metamaterials," Phys. Rev. B 81, 235111 (2010).

${ }^{33}$ J. Zhou, Th. Koschny, M. Kafesaki, E. N. Economou, J. B. Pendry, and C. M. Soukoulis, "Saturation of the magnetic response of splitring resonators at optical frequencies," Phys. Rev. Lett. 95, 223902 (2005). 